\title{
How to Optimise Cognitive Behaviour Therapy (CBT) for People with Autism Spectrum Disorders (ASD): A Delphi Study
}

\author{
Debbie Spain $^{1} \cdot$ Francesca Happé $^{1}$
}

Published online: 14 December 2019

(c) The Author(s) 2019

\begin{abstract}
Children and adults with autism spectrum disorders (ASD) can benefit from cognitive behaviour therapy (CBT), yet the prevailing opinion is that this requires adaptations to accommodate commonly experienced socio-communication and neuropsychological impairments. There are, however, no empirically-derived guidelines about how best to adapt standard practice. In a three round Delphi survey, we asked expert clinicians and clinical-researchers, based in England, about how to optimise the design, delivery and evaluation of CBT for people with ASD. Of 50 people approached, 18 consented to take part in Round 1, nine in Round 2 and eight in Round 3. Using a five-point scale, participants rated the degree to which 221 statements-pertaining to the referral process, assessment, engagement, formulation, goal setting, therapy structure, interventions and techniques, homework, outcome measurement, managing endings and therapist attributes-were integral to CBT. The consensus was that 155 statements represented essential or important components of CBT. Adaptations to the structure and process of therapy were consistently endorsed, and an individualised formulation-derived approach was favoured when deciding upon which interventions and techniques to offer. Further studies are needed to clarify if adapted CBT is associated with improved treatment outcomes and acceptability.
\end{abstract}

Keywords Autism spectrum disorders (ASD) · Cognitive behaviour therapy (CBT) • Delphi survey

Debbie Spain

debbie.spain@kcl.ac.uk

$1 \quad$ MRC Social, Genetic and Developmental Psychiatry Centre, Institute of Psychiatry, Psychology \& Neuroscience, King's College London, De Crespigny Park, PO Box 80, London SE5 8AF, UK 


\section{Introduction}

Autism spectrum disorder (ASD) is a neurodevelopmental condition, of childhood onset, characterised by socio-communication impairments and engagement in restricted interests, rituals and routines (American Psychiatric Association 2013). Children and adults with ASD experience high rates of comorbid mental health conditions, in particular, anxiety disorders, obsessive compulsive disorder (OCD) and depression (Russell et al. 2016; Simonoff et al. 2008; Wigham et al. 2017). They also commonly present with transdiagnostic characteristics (i.e. features occurring across psychological disorders), including an Intolerance of Uncertainty (IoU; Wigham et al. 2015), alexithymia (Kinnaird et al. 2019) and emotion dysregulation (Mazefsky et al. 2013). Causal and maintaining mechanisms for comorbidities are multi-factorial, and predominantly comprise psycho-social factors, possibly underpinned by neurobiological causes. Comorbidities exacerbate functional and social impairment, increase carer burden and impede quality of life (QoL) for people across the lifespan (Murphy et al. 2018). There is, therefore, an impetus for clinicalresearchers and service commissioners to develop and make available accessible and effective interventions.

In people without $\mathrm{ASD}$, cognitive behaviour therapy (CBT) is usually the recommended treatment of choice for anxiety disorders and depression (Clark 2011), and this has a strong evidence base (e.g. Hofmann et al. 2012; James et al. 2013). CBT is a short-term goal-focused 'talking therapy', based on several central premises: (1) there are interdependent relationships between what and how we think, how we feel physiologically and emotionally, and what we do; (2) unhelpful thoughts and thinking styles and particular coping strategies can indirectly perpetuate negative affect; and (3) negative affect and physiological anxiety and arousal can reinforce the use of less helpful responses and encourage negative thoughts and ways of thinking. In CBT, people are supported to make sense of the links between various aspects of presenting difficulties and develop alternative ways of thinking about, and responding to, real or perceived distressing stimuli (Kennerley et al. 2016).

Historically, CBT was primarily considered suitable for people with overt attributes suggestive of psychological-mindedness. Seminal work by Safran et al. (1993) concluded that patients most likely to benefit from short-term psychological interventions would have mild to moderate short-lived problems, a solution-focused stance, optimism and motivation to change, good insight into their thoughts and feelings as well as the ability to discriminate between these, and the capacity for reciprocity. Their work informed development of the Suitability for Short-Term Cognitive Therapy Rating Scale (SRS), and a handful of studies have reported significant associations between this and CBT outcomes in adults with depression or anxiety (Myhr et al. 2007; Renaud et al. 2014).

These criteria are, however, somewhat restrictive. Many people without ASD referred for CBT-either via the Improving Access to Psychological Therapies (IAPT) program in England or to psychological therapies services more widelyhave more complex presentations. This may be due to multi-morbidity, chronicity of symptoms, poor response to mono-treatment or difficulties with developing 
adaptive relationships (e.g. due to adverse social experiences). There are also significant clinical and ethical issues associated with refusing people psychological interventions because of their symptoms, interpersonal style or circumstances. Thus, in recent years, there have been more concerted efforts to adapt CBT for people presenting with varying degrees of clinical complexity, with good outcomes reported for adults with psychosis (Lincoln and Peters 2019), bipolar affective disorder (Chiang et al. 2017) and co-occurring physical and mental health conditions (e.g. Kew et al. 2016).

Traditionally, there have also seemed to be reservations about offering CBT to people with ASD (Moree and Davis 2010). Indeed, difficulties with accessing health services, including evidence-based psychological interventions, are commonly reported (e.g. Murphy et al. 2018) and generic service provision is seldom adapted or tailored for the needs of people with autism (National Institute for Health and Care Excellence; NICE 2012). This has resulted in, or coincided with, the development of specialist ASD services (e.g. Davidson et al. 2015), yet obtaining health authority funding for these can prove hugely difficult and there are long waiting lists for assessment and treatment.

Concerns about the suitability and acceptability, and thus effectiveness, of CBT for people with ASD may be attributable to several reasons. Core socio-communication characteristics, for example, have been hypothesised to hamper the development of a reciprocal therapeutic alliance; a fundamental mediating mechanism for psychological therapy effectiveness (Wampold 2015). Common neuropsychological impairments have also been proposed to impede engagement. Difficulties with introspection and interoception (Dubois et al. 2016; Kinnaird et al. 2019) may reduce awareness and accurate labelling of physiological sensations and emotions; an important component of psychological assessment and treatment. Impairments in theory of mind (ToM), perspective taking, generativity, cognitive flexibility and central coherence (Baron-Cohen et al. 2001; Brunsdon and Happé 2015) may affect the ability to identify and consider alternative possibilities (e.g. in terms of thoughts, beliefs and behaviours, or causes and outcomes of situations); again, integral to psychological interventions and techniques. More practically, sensory sensitivities (Koenig and Rudney 2010) and impairments in executive functioning (Tsatsanis 2014) may moderate information processing during appointments and when attempting tasks between sessions.

Yet conversely, there are several aspects of CBT that would suggest this could be a suitable approach for people with ASD, above and beyond other therapeutic modalities, if adapted to accommodate characteristics outlined above. The formulaic and methodical nature of CBT generally, and individual sessions specifically, can resonate well with people with ASD (e.g. due to preferences for order and predictability). Rather than the non-directive conversational styles characteristic of psychotherapy, discussions in CBT tend to be semi-structured and detail-focused (Kennerly et al. 2016); potentially more understandable for people with ASD who often have difficulties with abstract concepts. Developing a shared diagrammatic formulation of presenting difficulties can help to make these seem more contained. Individualised goals are well defined and these are tackled using a graded and collaborative approach. Finally, the emphasis on practice and application of specific interventions 
and techniques to multiple situations (e.g. graded exposure), enhances the possibility for generalisation of skills.

Reviews and meta-analyses indicate that the empirical evidence base for CBT for people with ASD has been developing more systematically since the late 1990s, although most studies have recruited children and adolescents. The evidence is encouraging. Case study and case series data suggest that CBT is an effective treatment for mental health symptoms, including low mood and self-harm (Hare 1997), anxiety disorders (Cardaciotto and Herbert 2004) and obsessive compulsive disorder (OCD; Reaven and Hepburn 2003). Results from randomised controlled trials (RCTs) suggest that CBT for anxiety and OCD can be more effective than treatment as usual and wait list controls and comparable to comparators (e.g. anxiety management; Weston et al. 2016). There is also preliminary evidence that CBT interventions and techniques are effective for transdiagnostic symptoms, including IoU (Rodgers et al. 2017), poor emotion regulation (Scarpa and Reyes 2011) and social skills impairments (Ung et al. 2015).

A consistent theme across studies is that the structure, process and content of CBT have been adapted to make this more accessible for participants, echoing broad recommendations outlined in the NICE guidelines for young people and adults with ASD (NICE 2011b, 2012). Narrative reviews of modifications of CBT in ASD intervention studies conclude that these have commonly included: (1) changing the number and duration of sessions; (2) using simple, concrete methods of conveying information; (3) doing more preparatory work (e.g. to enhance emotional literacy); (4) upskilling participants (e.g. to develop techniques applicable to multiple domains, such as problem-solving skills); (5) incorporating 'special interests' into treatment; (6) repeating tasks and techniques; (7) involving a co-therapist (e.g. a parent); and (8) liaising with others (e.g. teachers) (see Moree and Davis 2010; Walters et al. 2016).

While health services are mandated to provide needs-led interventions (e.g. HM 2009; NICE 2011b, 2012), there have been no formal efforts to operationalise which adaptations are more necessary for enhancing the accessibility of CBT for people with ASD. As the evidence base in this field grows, we need to better understand integral components of treatment.

\section{Study Aims}

The aims of this study were to establish and synthesise expert opinion about the design, delivery and evaluation of CBT for people with ASD, living in England, in order to develop consensus guidelines about good practice.

\section{Method}

\section{Study Design}

The study used a Delphi survey method; an iterative process for 'achieving convergence of opinion' from groups (Dalkey and Helmer 1963). Delphi studies are 
conducted frequently in health research, primarily to gather together the views of expert clinicians about poorly understood or under-evidenced topics, with the overall aim of establishing consensus about what best practice 'should or could' constitute (Hsu and Sandford 2007). Delphi studies include a series of surveys, usually administered in three consecutive rounds. Each survey comprises a list of statements developed by study researchers, participants or both; participants are asked to rate the degree to which these are pertinent and important for the clinical area under investigation. After each round, participants receive a summary of the group's results. If consensus agreement is not reached about particular statements (e.g. because views are very divergent), these are re-rated in a subsequent round. There are several advantages to using this method of data collection. Participant burden is diminished: surveys are often sent out online so these can be completed when convenient. Sampling frames can also be wider than is typically the case in face-toface research; potential participants can be approached from a larger geographic area and from multiple settings. Additionally, group, rather than individual responses are shared, so participants can be open, without feeling under pressure.

\section{Sampling Frame}

We compiled a list of clinicians and researchers, based in England, who have contributed to research protocols, journal articles and/or the development of clinical services, specifically for people with ASD. The list was developed by: (1) searching systematically for literature (empirical studies and narrative reviews) about CBT and ASD and reviewing author details; (2) conducting a search online for national ASD services; and (3) asking colleagues if they were aware of expert clinical-researchers working in the field. We restricted the sampling frame to England, as there are distinctions in the provision of clinical services across countries. We approached 50 potential participants, who collectively, worked at 24 sites (eight NHS Trusts, two health authorities and 14 universities).

\section{Survey Development}

Topics for the initial survey were generated by reviewing the following sources: (1) NICE guidelines for ASD (NICE 2011b, 2012) and CBT (NICE 2011a); (2) guidelines pertaining to the provision and supervision of CBT (Blackburn et al. 2001; IAPT 2007, 2018; Roth and Pilling 2008); (3) guidelines and standards about ethical conduct in clinical practice (BABCP 2017; BPS 2018; NMC 2015); (4) suggested CBT suitability criteria (Safran et al. 1993); (5) systematic reviews and opinion papers about CBT for people with ASD (including Anderson and Morris 2006; Rossiter and Holmes 2013; Spain et al. 2015; Ung et al. 2015; Weston et al. 2016); (6) Delphi studies focusing on CBT with other clinical populations (Morrison and Barratt 2010); and (7) clinical experience. Synthesis of the literature indicated that there were 11 key components of CBT to include in the initial survey: (1) the referral process; (2) assessment; (3) engagement; (4) formulation; (5) goal setting; (6) therapy 
structure; (7) interventions and techniques; (8) homework; (9) outcome measurement; (10) managing endings; and (11) therapist attributes. Notes were refined into summary statements and an online survey was developed.

\section{Measurement Scale}

Following well established guidelines for Delphi surveys (see Langlands et al. 2008), statements could be rated on a 5-point Likert scale: (1) essential; (2) important; (3) do not know / it depends; (4) unimportant; and (5) do not include.

\section{Usability Testing}

Initial piloting of the survey content and format with two clinical psychologists and one nurse consultant resulted in the rewording of a few statements for clarity or brevity and addition of a few statements. Piloting of the survey software with three post-graduate researchers, resulted in a modification to the method of navigation between survey sections.

\section{Ethical Approvals}

The study was granted approvals by the Research Ethics Committee (REC REF 14 0558), local R\&D leads and Heads of Department. Informed consent was obtained from all participants.

\section{Procedure}

Recruitment took place between July 2017 and September 2018. The survey comprised three rounds; each had the same process, with similar formatting. Participants were contacted by email with a unique log in to the survey site (hosted by Qualtrics), accessible via a computer, tablet or smartphone. The initial section of the survey outlined study information and consent. Thereafter, survey statements were listed in 11 sections (under headings as outlined above), with additional free text options. Responses were autosaved so the survey could be completed at several junctures. Round 1 also included some general demographic questions (see below). Rounds two and three included statements from the previous round if these required re-rating and new statements suggested by participants.

\section{Data Analysis}

We summarised descriptive information about participant demographic characteristics. We then calculated the percentage of participants who endorsed each option per 
statement in Excel. Based on the guidelines by Langlands and colleagues (2008), we adopted the following parameters: (1) statements rated as either essential or important by approximately $\geq 80 \%$ of participants were considered an integral component of CBT; (2) statements attaining a consensus rating of approximately $60-79 \%$ were re-rated in the following round (but only once more); and (3) statements that did not meet these criteria were subsequently excluded. Since different numbers took part in each round, percentages sometimes differed slightly to reflect percentages to the nearest whole person.

\section{Results}

\section{Round 1}

Of 50 individuals approached, 18 completed Round 1 (a 36\% response rate). (See Fig. 1). Participants worked at five NHS Trusts and six universities. In terms of geographic location, ten participants worked within Greater London, four in the West of England, two in the South of England and two in the North of England. Sixteen participants (66\% women) were clinical psychologists, one a nurse, and one an academic. Post-qualification clinical experience of work with people with ASD ranged from 3-32 years (median 11 years). Participants worked in inpatient, community and outpatient departments, and primary through to tier four settings, and many were clinical-academics. Seven participants worked with young people, six with adults, and five with people across the lifespan.

The Round 1 survey comprised 144 statements. Of these, 88 were deemed essential or important with approximately $80 \%$ consensus (rated as such by $\mathrm{n} \geq 14$ ), 19 statements required re-rating as these attained $60-79 \%$ consensus (defined as agreement between 11-13 participants), and 37 statements were excluded as there was a lack of consensus amongst $59 \%$ of participants or fewer $(n \leq 10)$. Synthesis of participants' free text responses resulted in a further 70 statements.

\section{Round 2}

Nine participants completed the Round 2 survey, which comprised 89 statements (19 that required re-rating and 70 that had been generated by participants). In this round, 58 statements were described as essential or important with approximately $80 \%$ consensus (rated as such by $\mathrm{n} \geq 7$ ), 10 required re-rating as these attained agreement by $60-79 \%$ of the sample $(n=6)$ and the remainder were excluded as these were rated similarly by 5 or fewer participants. A further seven statements were suggested. 


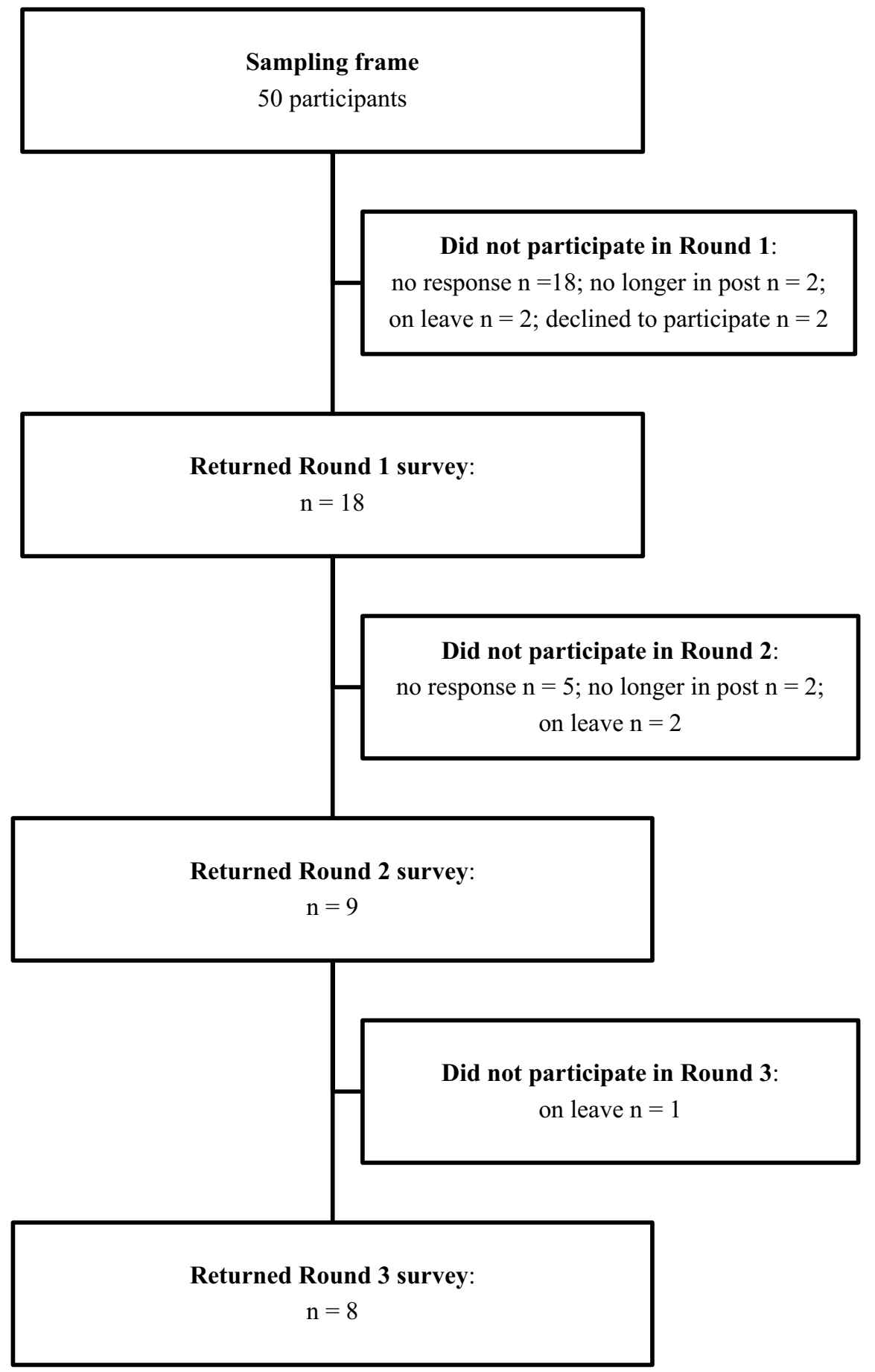

Fig. 1 Respondent flow chart 


\section{Round 3}

The Round 3 survey, which had 17 statements (10 that required re-rating and seven new statements generated by participants), was completed by eight participants. Of these, nine were deemed essential or important by 6 or more participants, and eight were excluded due to a lack of consensus $(n \leq 5)$.

\section{Summary of Results}

See Table 1 for an overview of the statements, categorised by theme. We found that the statements $(n=221)$ pertained to three broad categories of approaches and attributes: (1) generic; (2) ASD-specific; and (3) other-focused. Overall, 155 statements were deemed essential or important aspects of CBT for people with ASD (outlined in Table 2), and 66 statements were excluded (the list is available on request). No statements attained a consensus rating of 'do not include'.

\section{Discussion}

Previous research indicates that clinicians and triallists adapt their standard practice to enhance the suitability and acceptability of CBT for people with ASD. Yet there are no guidelines outlining which modifications might be most widely accepted as useful. In a three round Delphi survey, we established consensus views from expert clinicians and researchers in England, about the optimisation of CBT for this clinical population.

Reflecting wider opinion, participants agreed that CBT is an appropriate treatment choice for people with ASD (Gaus 2011; Moree and Davis 2010). Analysis of

Table 1 Summary of themes and items per survey round

\begin{tabular}{lllllll}
\hline Themes & \multicolumn{2}{l}{ Data collection period } & & \multicolumn{2}{l}{ Final survey } \\
\cline { 2 - 3 } \cline { 6 - 7 } & Round 1 & Round 2 & Round 3 & & Included & Excluded \\
\hline Referrals & 0 & $4(4 \%)$ & $1(6 \%)$ & $2(1 \%)$ & $2(3 \%)$ \\
Assessment & $15(10 \%)$ & $22(25 \%)$ & $1(6 \%)$ & $28(18 \%)$ & $5(8 \%)$ \\
Engagement & $13(9 \%)$ & $14(16 \%)$ & $3(18 \%)$ & $24(15 \%)$ & $5(8 \%)$ \\
Formulation & $15(10 \%)$ & $10(11 \%)$ & $2(12 \%)$ & $19(12 \%)$ & $5(8 \%)$ \\
Goal setting & $8(6 \%)$ & $3(3 \%)$ & $1(6 \%)$ & $10(6 \%)$ & $1(2 \%)$ \\
Therapy structure & $18(13 \%)$ & $9(10 \%)$ & $1(6 \%)$ & & $14(9 \%)$ & $1015 \%)$ \\
Interventions / techniques & $31(22 \%)$ & $9(10 \%)$ & $1(6 \%)$ & & $12(8 \%)$ & $23(35 \%)$ \\
Homework & $9(6 \%)$ & $5(6 \%)$ & $2(12 \%)$ & $14(9 \%)$ & $2(3 \%)$ \\
Outcome measurement & $8(6 \%)$ & $7(8 \%)$ & $5(29 \%)$ & $5(3 \%)$ & $8(12 \%)$ \\
Managing endings & $7(5 \%)$ & $5(5 \%)$ & 0 & $9(6 \%)$ & $2(3 \%)$ \\
Therapist attributes & $20(14 \%)$ & $1(1 \%)$ & 0 & $18(12 \%)$ & $35 \%)$ \\
Totals & 144 & 89 & 17 & 155 & 66 \\
\hline
\end{tabular}




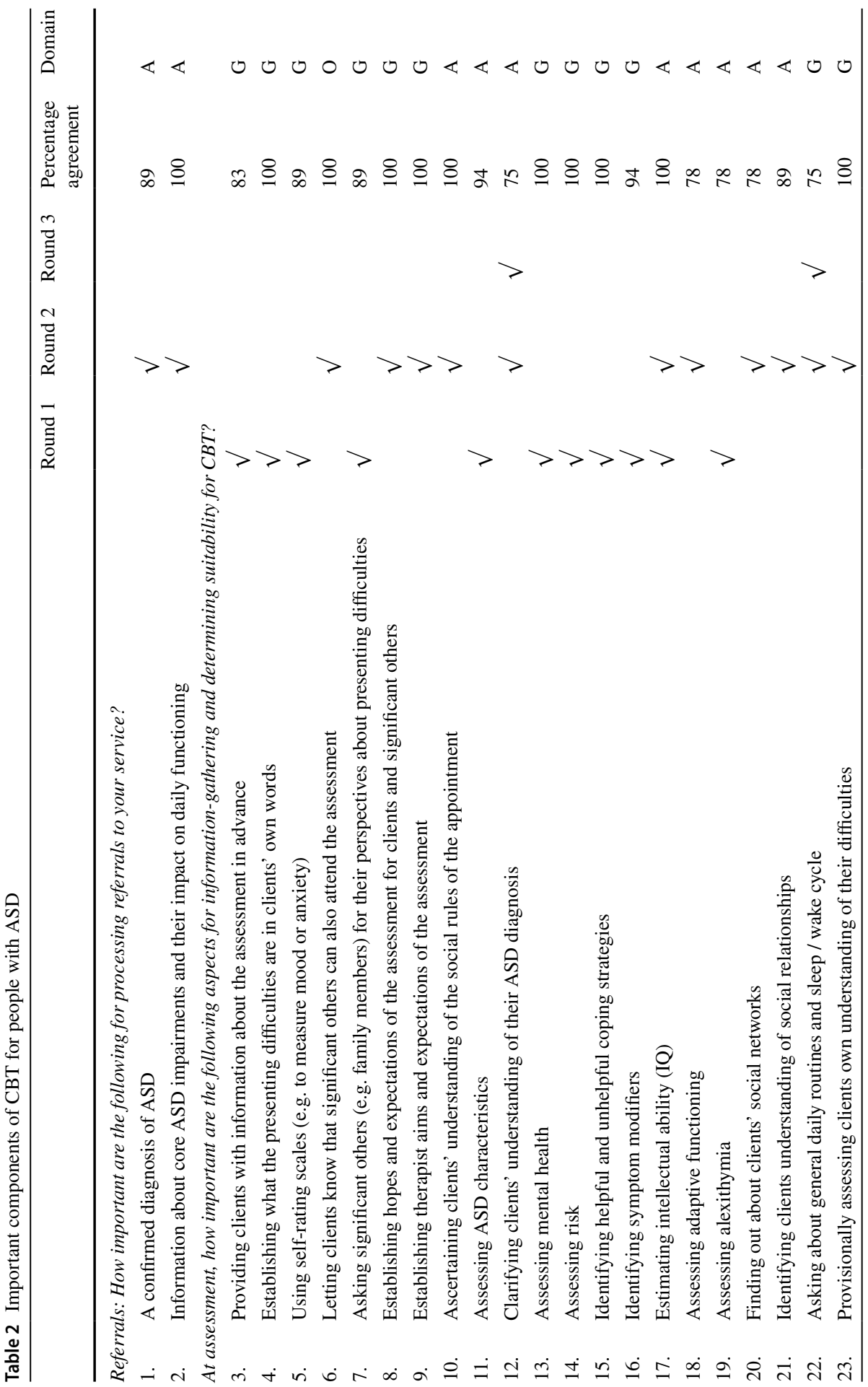




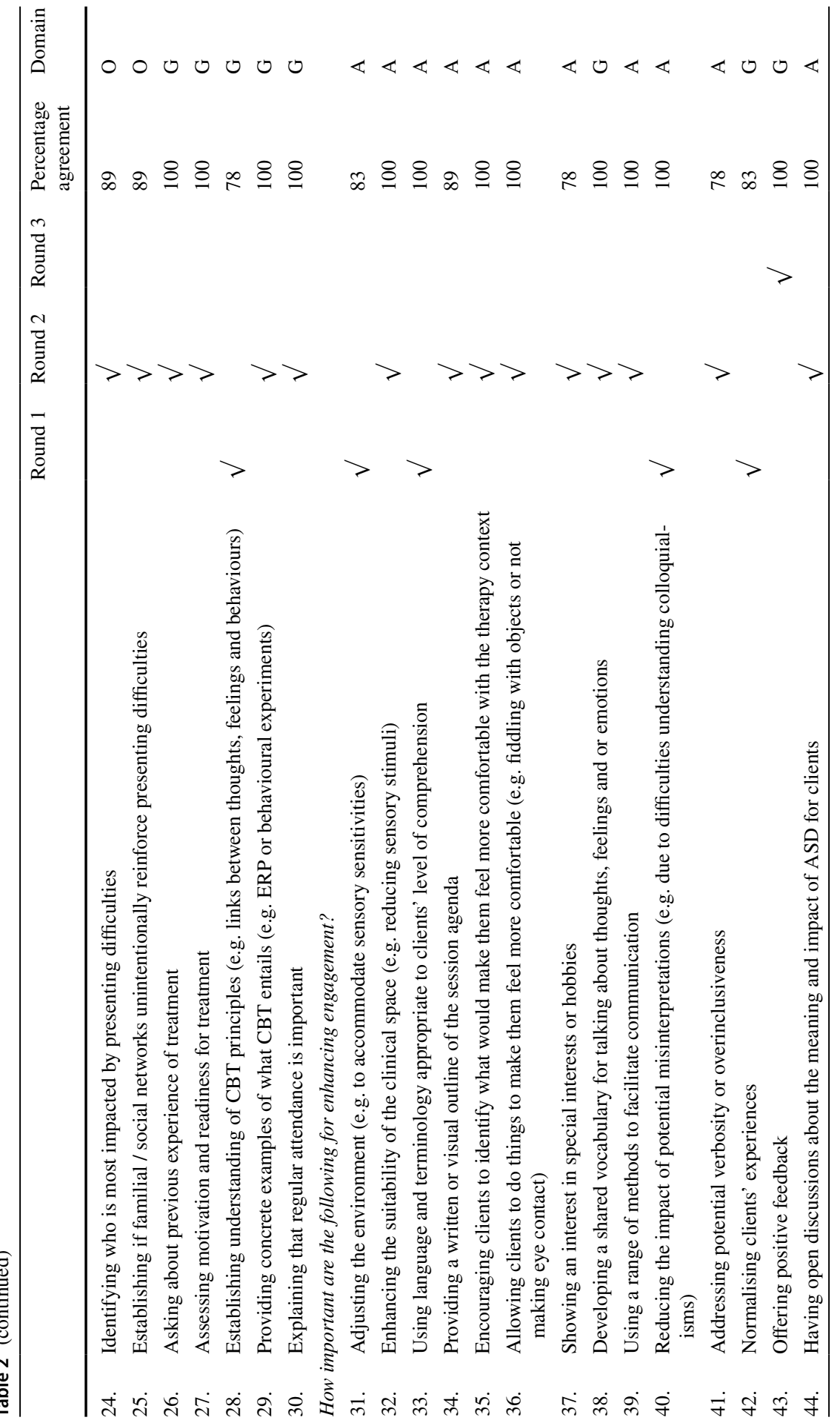




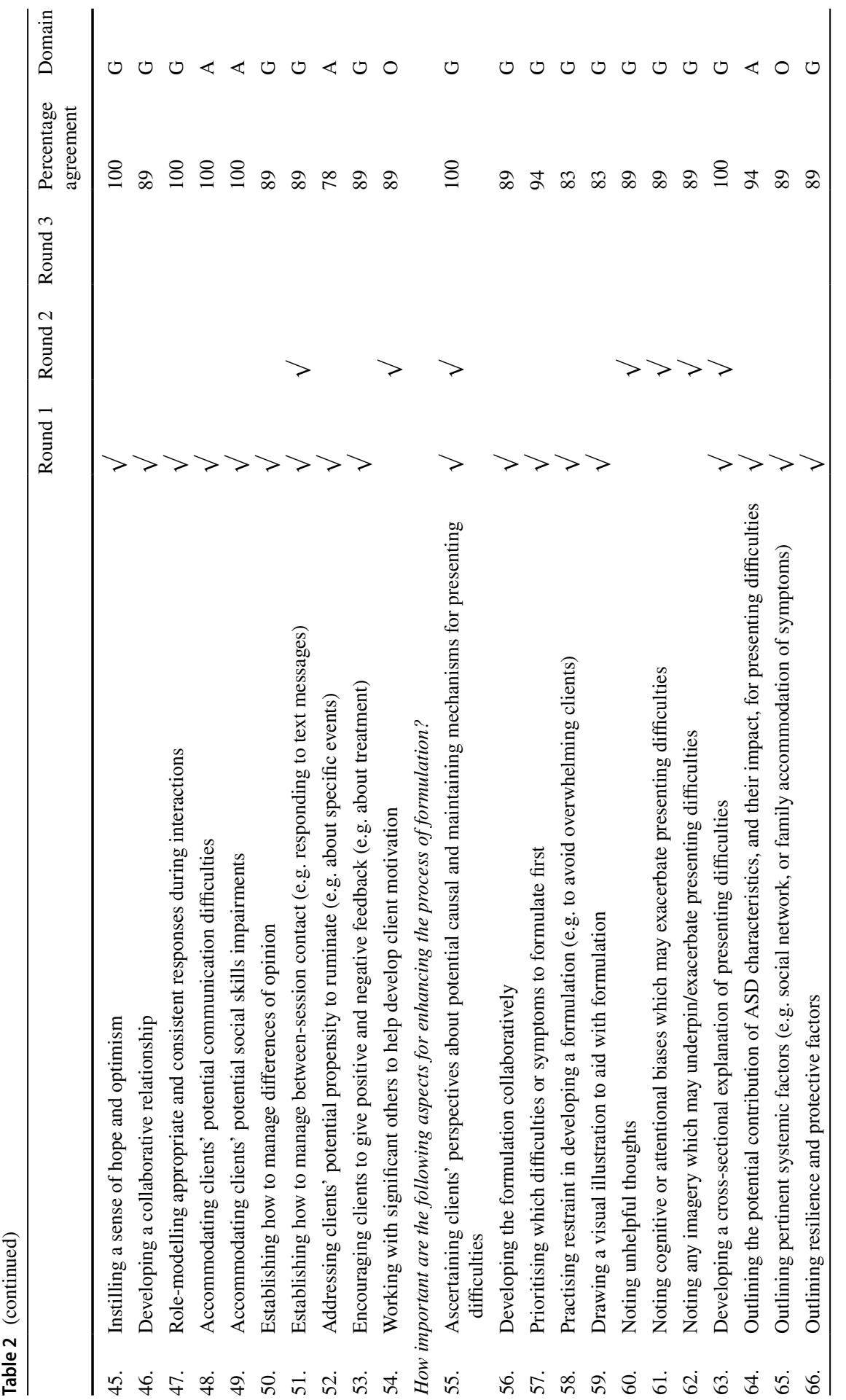




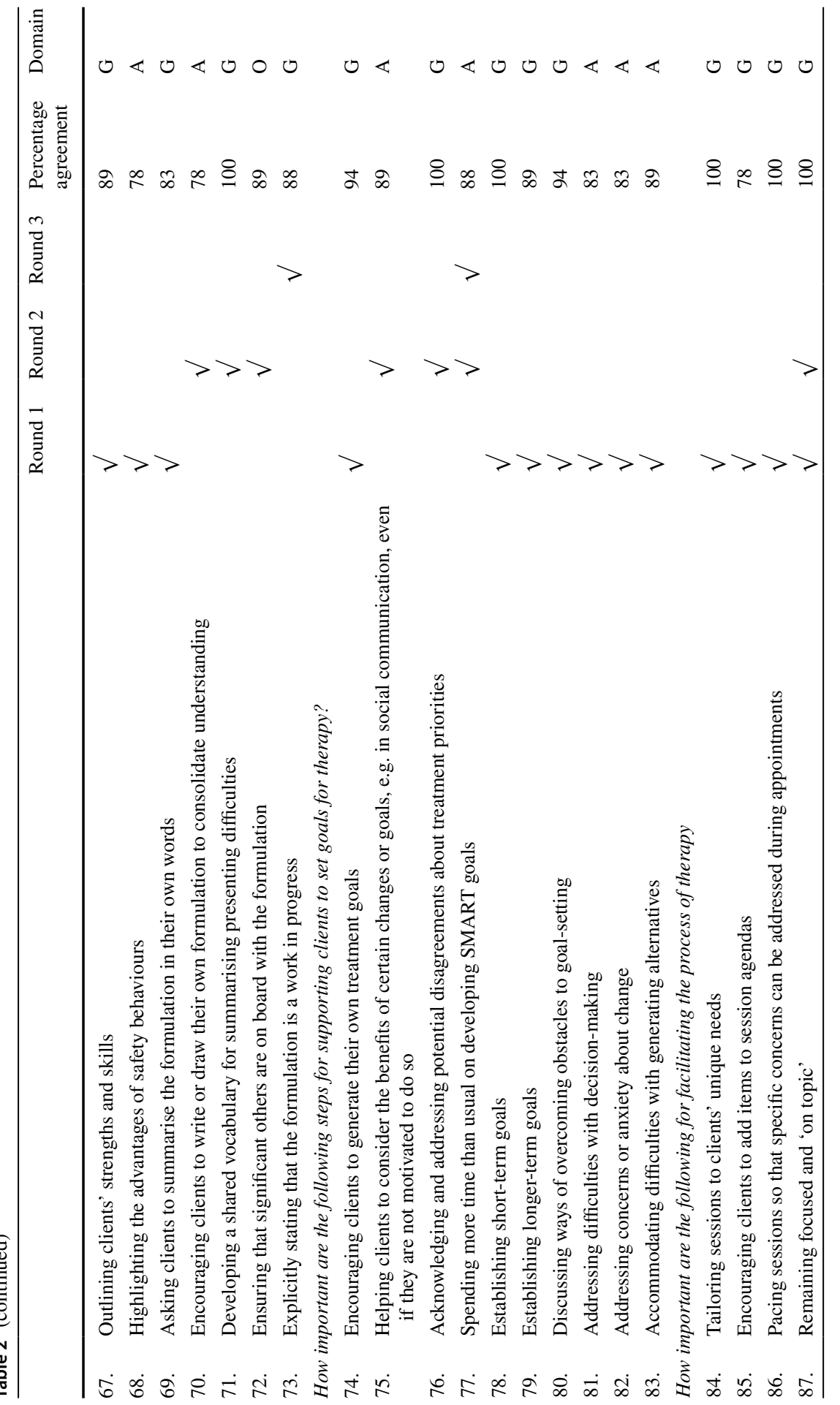




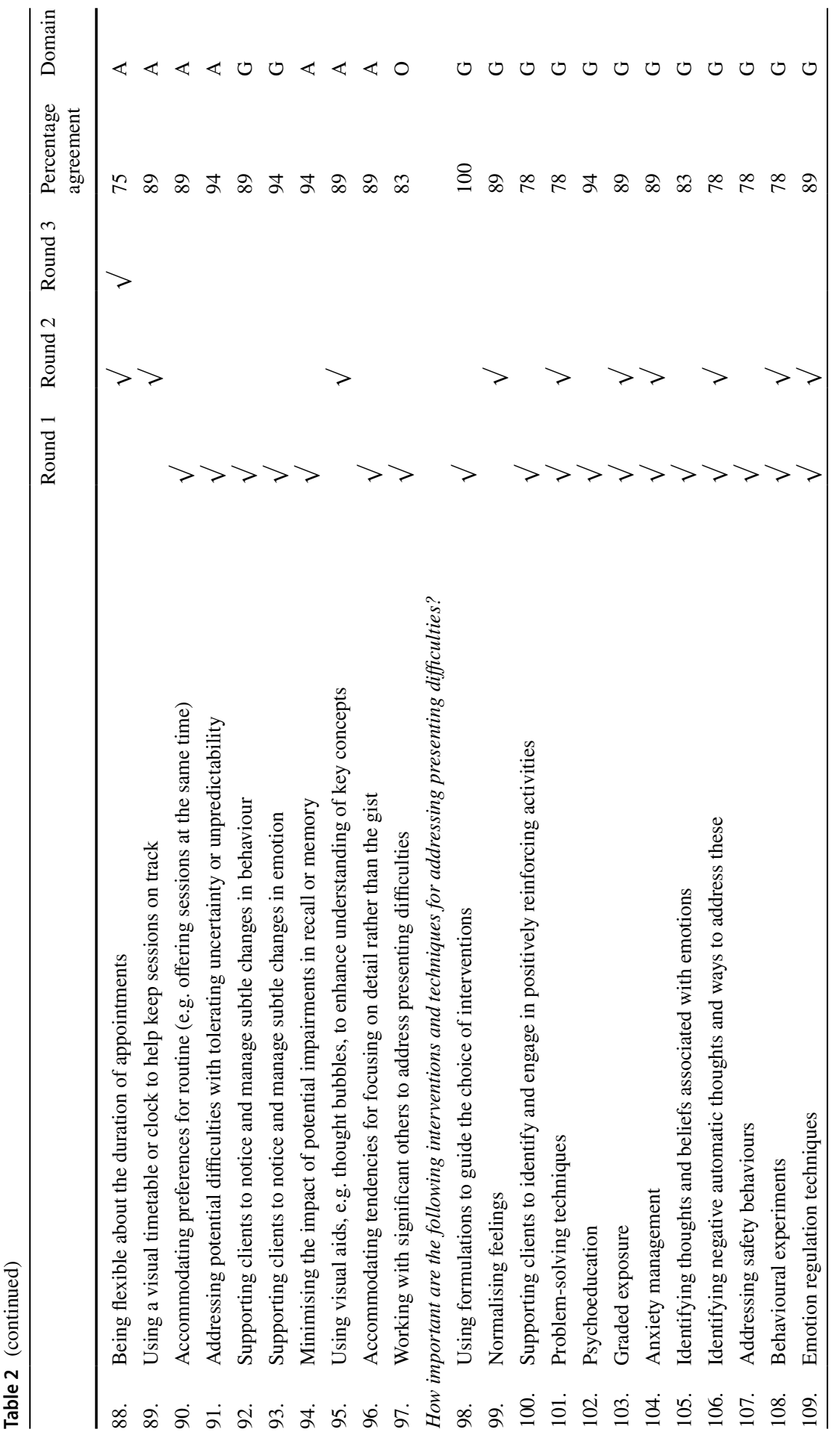




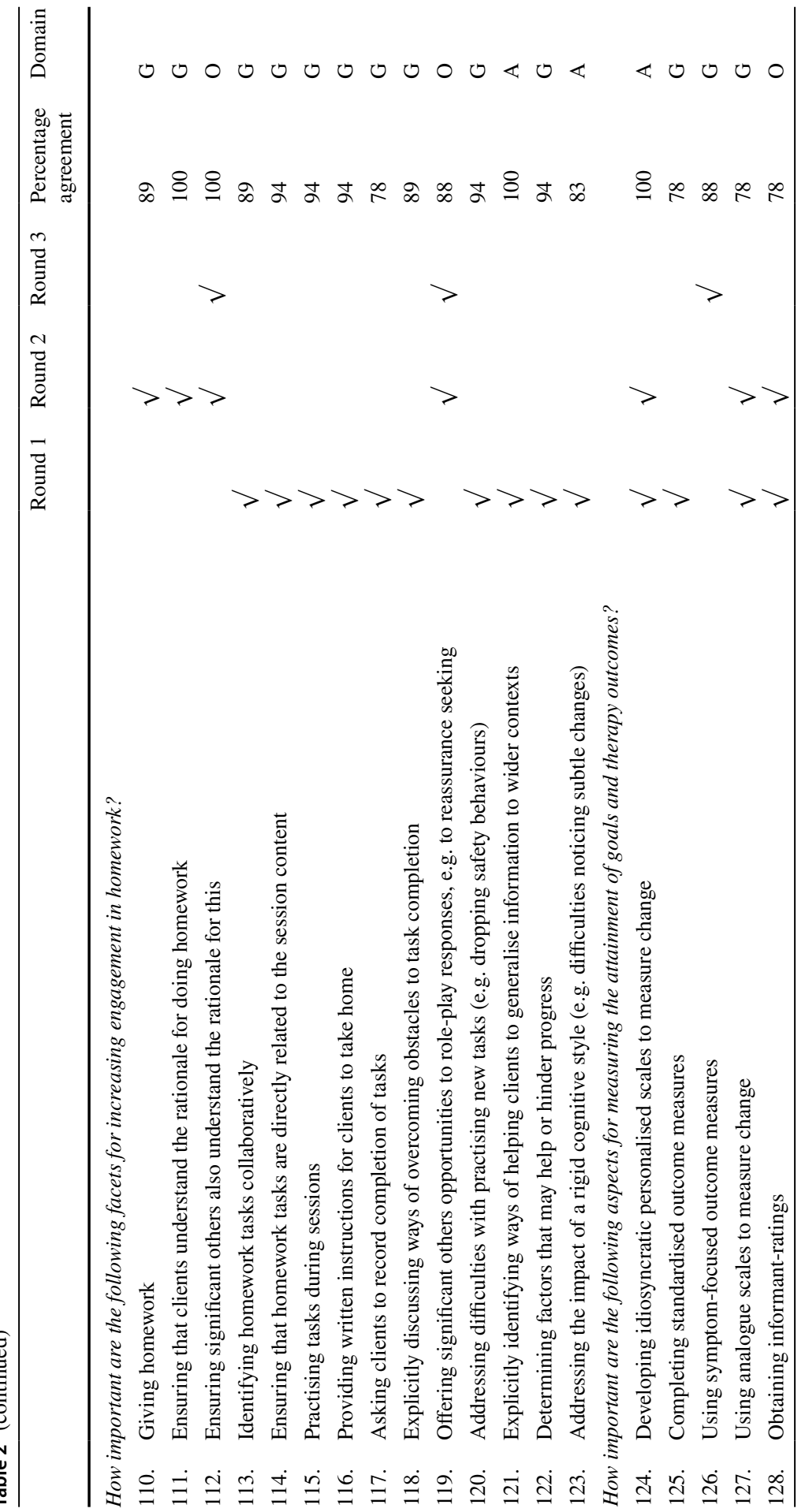




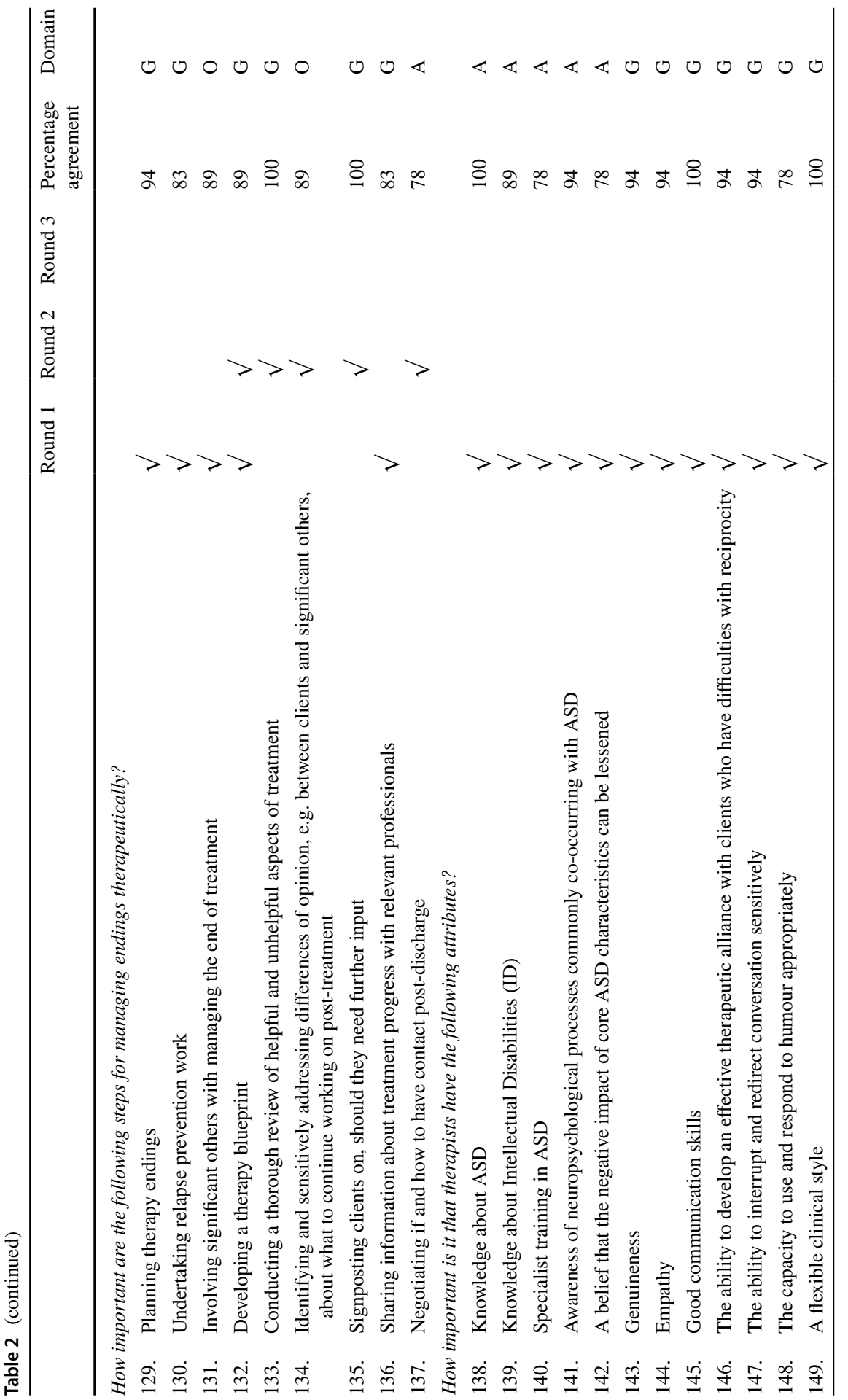




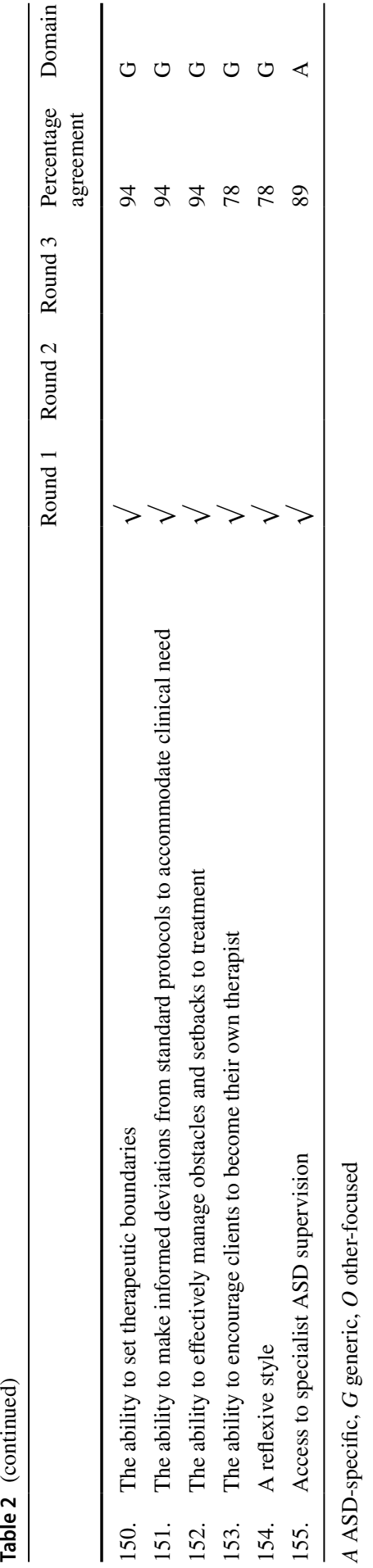


free text responses suggested that particular factors potentially render CBT unsuitable for some people, such as aspects of the clinical presentation, attitudes and motivation, risk and practical constraints (summarised in Table 3). Importantly, these factors apply to people with and without neurodevelopmental conditions. Moreover, they are by and large fluid, rather than static constructs. The clinical implication is that suitability or readiness for CBT is best evaluated periodically: some people may not be ready to engage at the point of referral, yet may be more able and amenable at another time.

\section{Generic approaches and attributes}

Approximately half the statements included in the final survey represented generic approaches or attributes, that would be expected from therapists working with most clinical populations. These approaches included establishing situational, interpersonal, cognitive, behavioural and affective aspects of presenting difficulties, formulating links between these collaboratively, using strategies within and outside of sessions to encourage change, and enhancing confidence, autonomy and resilience. Some of the statements and particularly those about assessment and therapist attributes, are characteristic of many psychotherapeutic modalities (e.g. 'establishing what the presenting difficulties are in clients' own words'). Yet a substantial proportion of these are specific to CBT (e.g. 'developing a cross-sectional explanation of presenting difficulties'). This suggests that people with ASD can make use of and benefit from the fundamental facets that delineate CBT from other modalities.

Importantly, a number of the generic approaches that were excluded pertained to specific interventions and techniques. Feedback from participants demonstrated that although it was possible to rate the importance of transdiagnostic CBT interventions (e.g. 'graded exposure' and 'addressing safety behaviours'), it was less meaningful to do so, for specific ways of working (e.g. 'imaginal exposure' and 'ERP'), as these are based on the formulation and treatment goals. In a comparable Delphi survey, that sought consensus expert views about CBT for people with psychosis, Morrison and Barratt (2010) reported that their participants had similar concerns. They

Table 3 Factors that may render CBT unsuitable for people with ASD

\begin{tabular}{ll}
\hline Clinical presentation & Attitudes and motivation \\
\hline Age, specifically very young children & $\begin{array}{c}\text { Egosyntonic explanation } \\
\text { for symptoms } \\
\text { Poor insight }\end{array}$ \\
$\begin{array}{ll}\text { Cognitive capacity: moderate to severe ID } \\
\text { If another intervention is indicated, e.g. medication }\end{array}$ & Poor motivation \\
Current excessive alcohol or substance use & Practical constraints \\
\hline Risk & Lives very far from clinic \\
\hline Significant risk to self & Unable to travel \\
Significant risk to others & \\
Fluctuating risk & \\
\hline
\end{tabular}


also noted that other patient-related factors, such as 'engagement' and 'readiness to change' could mediate appropriateness of some interventions, and that prefacing some statements with additional information might enhance their validity.

\section{ASD-Specific Adaptations to CBT}

Data analysis indicated that $40 \%$ of approaches and attributes deemed integral were ASD-specific. These primarily concerned adaptations to accommodate core ASD characteristics (e.g. 'ascertaining clients' understanding of the social rules of the appointment'), as well as co-occurring neuropsychological impairments (e.g. 'addressing difficulties with decision-making'). Moreover, there were three main types of adaptation: (1) those that denoted an addition to standard practice (e.g. 'allowing clients to do things that make them feel more comfortable such as fiddling with objects or not making eye contact'); (2) those that were omitted from standard practice (e.g. 'noting core beliefs'); and (3) those that constituted modifications to conventional approaches (e.g. 'using a range of methods to facilitate communication'). Importantly, participant responses indicated that adaptations are pertinent for CBT with young people and adults with ASD and so these are therefore unlikely to merely reflect age-related differences in treatment protocols.

A general trend in the data suggested that decision-making about types of adaptations incorporated or omitted might be based on a number of factors. For example, good knowledge and understanding of ASD, coupled with a sense that impairing symptoms and circumstances can be improved, could be linked to more focused attempts to engage patients in the manner they felt comfortable with as well as creativity in the process of formulation and use of techniques. A more thorough assessment of the nature and impact of core characteristics, co-occurring neuropsychological impairments and meaning-making of these, could inform methods for enhancing communication during and outside of sessions. Also, therapists' capacity for flexibility and responsivity to patients' presentations could be associated with attempts to make the structure of sessions better tailored to patients' needs.

Modifications to standard CBT approaches endorsed here are consistent with those described by clinicians and researchers elsewhere (e.g. Anderson and Morris 2006; Moree and Davis 2010; Walters et al. 2016). Broadly speaking, prior research has indicated that a range of general adaptations are needed to the structure and process of CBT. However, the findings reported here add to the literature by outlining the more specific adaptations that are potentially needed at each stage of the treatment process. Moreover, historically, there has been debate about the degree to which cognitive techniques might be suitable for people with ASD given common impairments in ToM and perspective-taking. Consensus views here, however, suggest that these techniques, are in fact an important component of CBT.

Clinically, it appears that enhanced knowledge and understanding of ASD and associated characteristics and impairments are key to informing choices about when and how to adapt conventional CBT approaches. The implication is that service providers and team managers should ensure staff have access to relevant training. This matches the recommendations outlined in the Transforming Care initiative (HM 
2019), which aims to enhance the knowledge and skills of frontline staff working with people with ASD and/or ID. Our study findings also reinforce the importance of asking patients directly (and also potentially, their significant others) about their expectations, preferences and difficulties. Doing so periodically, can help clinicians to choose how and what to adapt.

It is possible that specialist clinical supervision (also deemed important by participants) could aid health professionals to think through the types of adaptations that each patient might benefit from. Recent studies of CBT therapists working with other clinical populations have concluded that supervision should incorporate opportunities for discussion, reflection, knowledge acquisition and skills rehearsal (Bennett-Levy et al. 2009). In our experience, we have found that individual and group ASD-focused supervision can prove beneficial. The latter may be more resource-efficient, especially if clinicians work in generic psychological therapies services with a small caseload of patients with ASD.

\section{Working with Significant Others}

The majority of participants — working with people of all ages — agreed that significant others should be involved in treatment, and other-focused approaches represented approximately $10 \%$ of statements in the final survey. These included considering others during the process of assessment (e.g. clarifying the extent to which they may be affected by, or contribute indirectly to, presenting difficulties), facilitating emotionally salient conversations between patients and parents (e.g. about differing priorities for treatment and post-discharge), and asking the latter to take on a more 'active' role (e.g. supporting task completion outside of sessions and changing responses to (safety) behaviours). Interestingly, 'family-focused work' was excluded from the final survey and it may be that participants viewed this more specifically as a systemic rather than a CBT intervention.

In young people without ASD, parental involvement in CBT has become increasingly common, yet data are conflicting about whether this gleans more favourable outcomes compared with child-alone treatment (Thulin et al. 2014). Preliminary evidence indicates that parental involvement is beneficial in CBT for children and adolescents with ASD (Cappadocia and Weiss 2011), but to our knowledge, there have been no head-to-head comparisons with active comparators. Conversely, it is unusual for significant others to be involved in CBT to such a great extent with non-ASD adults and this has not constituted a major element of published empirical treatment studies recruiting adults with ASD (Spain et al. 2015). Yet, many people with ASD, across the lifespan, benefit from additional support, for example, due to the impact of core ASD characteristics, neuropsychological impairments and mental health conditions. Thus, clinically, it is important to find out how much involvement patients would like other people in their lives to have. More practically, not all adults with ASD have contact with family and their social network is often smaller than they would like. This means that for some patients, in vivo work in the community may be particularly important to aid with generalising of skills. 


\section{Outcome Measurement}

Participant consensus indicated that standardised outcomes measures (e.g. generic and disorder-specific self-report questionnaires) should be augmented with personalised and more visual means, such as idiosyncratic symptom-focused or analogue scales; administered periodically rather than weekly. There are potentially several reasons for this. The utility of standardised self-report measures of (mental) health and wellbeing for people with ASD, has not yet been established (e.g. Brugha et al. 2015). This is partly because the content (i.e. items or questions) require further validation empirically, and also, as completion of these can prove difficult for people with ASD (e.g. due to impairments in introspection and cognitive characteristics including perseveration and rigidity). Individualised measures may, therefore, resonate more strongly and capture more accurately the presenting difficulties of people with ASD. It is also possible that developing outcome measures with patients enhances engagement and the 'buy in' to treatment.

Clinically, therapists may need to collaborate with patients to identify preferences for how difficulties are operationalised and measured (e.g. words and/or images), how frequently to complete these and where to do so (e.g. at home or with therapist support). This may require extra sessions, but the resources incurred could be offset by increased measurement sensitivity and specificity. Also, idiosyncratic scales may have utility for other strands of the mental health care pathway, in a similar way to a 'health passport' (NICE 2012). For example, individualised measures of mood states and behavioural indicators of affect, may be equally informative for a psychiatrist or community psychiatric nurse.

\section{Study Limitations}

There are several study limitations. We restricted our sampling frame $(n=50)$ to experts working in England. While we sought out clinicians and researchers who have contributed to published work and service innovation, we did not reach all professionals with substantial expertise. It is also probable that the provision of CBT for people with ASD differs slightly outside of England, such as in terms of eligibility criteria for services and availability of CBT, so study findings may not be generalisable to some other settings. The participant response rate was lower than we hoped. This may be attributable to several factors, including time, length of the initial survey and time lags between rounds, meaning that some participants from previous rounds were no longer in post or on leave when subsequent surveys were circulated. Due to the sample size, we were unable to investigate whether the relative importance of aspects of CBT was age-specific, such as by comparing responses from participants working with children vs. adults, or whether participant responses varied according to experience. Statements were generated from empirical and clinical sources, but this process could have been enhanced with service-user involvement. Finally, we used the same measurement scale as many other Delphi studies, including having one rating encompassing both 'do not 
know' and 'it depends'. Yet as reported by several participants and elsewhere in the literature, these are not synonymous.

\section{Research Implications}

Clearly, the evidence-base for the utility, effectiveness and acceptability of CBT for people with ASD needs to be extended. Importantly, much of the intervention research to date has been obtained using quantitative methods. A next step is to garner more systematically, the views of people with ASD about what it is they hope to gain from CBT, which aspects of treatment they find more or less useful and why this is. Further studies are needed to clarify, more precisely, in what instances and how clinicians adapt their standard practice, and whether this differs according to knowledge, skills or experience, or service setting. Moreover, studies comparing adapted vs. standardised CBT approaches would provide clarity about which modifications are actually associated with improved outcomes.

\section{Conclusions}

Intervention research has lagged behind studies focusing on prevalence and correlates of co-occurring conditions in people with ASD. Yet the growing evidence base suggests that CBT can be beneficial. Very few studies have focused on moderating and mediating mechanisms: we do not yet know how, why or for whom CBT is more or less effective, nor whether there are particular adaptations that glean more favourable outcomes. The consensus view from our study participants suggested that many of the core facets, interventions and techniques integral to CBT are accessible and useful for people with ASD. However, there is a need to adapt the method of delivery, using flexibility and creativity. Further studies examining how best the structure, process and content can be modified for people with ASD are now warranted.

Acknowledgements DS was funded by a National Institute for Health Research (NIHR) Clinical Doctoral Research Fellowship between 2013 and 2018 (CDRF-2012-03-059). This paper presents independent research funded by the NIHR. FH is part funded by the NIHR Biomedical Research Centre at South London and Maudsley NHS Foundation Trust and King's College London. The views expressed are those of the author(s) and not necessarily those of the NHS, the NIHR or the Department of Health.

\section{Compliance with Ethical Standards}

Conflicts of interest The authors declare that they have no conflicts of interest.

Ethical Approvals Research ethics approvals were obtained to conduct the study (REC REF 14 0558).

Informed Consent Written informed consent was obtained from all participants.

Open Access This article is licensed under a Creative Commons Attribution 4.0 International License, which permits use, sharing, adaptation, distribution and reproduction in any medium or format, as long as you give appropriate credit to the original author(s) and the source, provide a link to the Creative Commons licence, and indicate if changes were made. The images or other third party material in this article 
are included in the article's Creative Commons licence, unless indicated otherwise in a credit line to the material. If material is not included in the article's Creative Commons licence and your intended use is not permitted by statutory regulation or exceeds the permitted use, you will need to obtain permission directly from the copyright holder. To view a copy of this licence, visit http://creativecommons.org/licen ses/by/4.0/.

\section{References}

American Psychiatric Association. (2013). DSM-V. APA.

Anderson, S., \& Morris, J. (2006). Cognitive behaviour therapy for people with Asperger syndrome. Behavioural and Cognitive Psychotherapy, 34, 293-303.

Baron-Cohen, S., Wheelwright, S., Hill, H., Raste, Y., \& Plumb, I. (2001). The "Reading the Mind in the Eyes" test revised version: A study with normal adults, and adults with Asperger syndrome or high functioning autism. Journal of Child Psychology and Psychiatry, 42, 241-251.

Bennett-Levy, J., McManus, F., Westling, B. E., \& Fennell, M. (2009). Acquiring and refining CBT skills and competencies: Which training methods are perceived to be most effective? Behavioural and Cognitive Psychotherapy, 37, 571.

Blackburn, I. M., James, I. A., Milne, D. L., Baker, C., Standart, S., Garland, A., et al. (2001). The revised cognitive therapy scale (CTS-R): Psychometric properties. Behavioural and Cognitive Psychotherapy, 29, 431-446.

British Association for Behavioural and Cognitive Psychotherapies (BABCP). (2017). Standards of conduct, performance and ethics. England: BABCP.

British Psychological Society (BPS). (2018). Code of Ethics and Conduct. UK: BPS.

Brugha, T. S., Doos, L., Tempier, A., Einfeld, S., \& Howlin, P. (2015). Outcome measures in intervention trials for adults with autism spectrum disorders; A systematic review of assessments of core autism features and associated emotional and behavioural problems. International Journal of Methods in Psychiatric Research, 24, 99-115.

Brunsdon, V. E. A., \& Happé, F. G. (2015). Exploring the "fractionation" of autism at the cognitive level. Autism, 18, 17-30.

Cappadocia, M. C., \& Weiss, J. A. (2011). Review of social skills training groups for youth with Asperger syndrome and high functioning autism. Research in Autism Spectrum Disorders, 5, 70-78.

Cardaciotto, L., \& Herbert, A. D. (2004). Cognitive behavior therapy for social anxiety disorder in the context of Asperger's syndrome: A single-subject report. Cognitive and Behavioral Practice, 11, $75-81$.

Chiang, K.-J., Tsai, J.-C., Liu, D., Lin, C.-H., Chiu, H.-L., \& Chou, K.-R. (2017). Efficacy of cognitivebehavioral therapy in patients with bipolar disorder: A meta-analysis of randomized controlled trials. PLoS ONE, 12, e0176849.

Clark, D. M. (2011). Implementing NICE guidelines for the psychological treatment of depression and anxiety disorders: The IAPT experience. International Review of Psychiatry, 23, 318-327.

Dalkey, Norman, \& Helmer, Olaf. (1963). An experimental application of the Delphi method to the use of experts. Management Science, 9, 458-467.

Davidson, C. J., Kam, A., Needham, F., \& Stansfield, A. J. (2015). No exclusions-developing an autism diagnostic service for adults irrespective of intellectual ability. Advances in Autism, 1, 66-78.

DuBois, D., Ameis, S. H., Lai, M. C., Casanova, M. F., \& Desarkar, P. (2016). Interoception in autism spectrum disorder: A review. International Journal of Developmental Neuroscience, 52, 104-111.

Gaus, V. (2011). Cognitive behavioural therapy for adults with autism spectrum disorders. Advances in Mental Health and Intellectual Disabilities, 5, 15-25.

Hare, D. J. (1997). The use of cognitive-behavioural therapy with people with Asperger syndrome: A case study. Autism, 1, 215-225.

HM Government. (2009). Autism Act. UK. http://www.legislation.gov.uk/ukpga/2009/15/contents.

HM Government. (2019). Transforming Care. UK. https://www.england.nhs.uk/wp-content/uploa ds/2017/02/model-service-spec-2017.pdf.

Hofmann, S. G., Asnaani, A., Vonk, I. J. J., Sawyer, A. T., \& Fang, A. (2012). The efficacy of cognitive behavioral therapy: A Review of meta-analyses. Cognitive Therapy and Research, 36, 427-440. 
Hsu, C. C., \& Sandford, B. A. (2007). The Delphi technique: Making sense of consensus. Practical Assessment, Research and Evaluation, 12, 1-8.

IAPT (2007). The competences required to deliver effective cognitive and behavioural therapy for people with depression and anxiety disorders. England: IAPT.

IAPT: National Collaborating Centre for Mental Health. (2018). The improving access to psychological therapies manual. England: National Collaborating Centre for Mental Health.

James, A. C., James, G., Cowdrey, F. A., Soler, A., \& Choke, A. (2013). Cognitive behavioural therapy for anxiety disorders in children and adolescents. Cochrane Database of Systematic Reviews., 6, CD004690.

Kennerley, H., Kirk, J., \& Westbrook, D. (2016). An introduction to cognitive behaviour therapy: Skills and applications. Beverly Hills: Sage.

Kew, K. M., Nashed, M., Dulay, V., \& Yorke, J. (2016). Cognitive behavioural therapy (CBT) for adults and adolescents with asthma. Cochrane Database of Systematic Reviews., 9, CD011818.

Kinnaird, E., Stewart, C., \& Tchanturia, K. (2019). Investigating alexithymia in autism: A systematic review and meta-analysis. European Psychiatry, 55, 80-89.

Koenig, K. P., \& Rudney, S. G. (2010). Performance challenges for children and adolescents with difficulty processing and integrating sensory information: A systematic review. American Journal of Occupational Therapy, 64, 430-442.

Langlands, R. L., Jorm, A. F., Kelly, C. M., \& Kitchener, B. A. (2008). First aid for depression: A Delphi consensus study with consumers, carers and clinicians. Journal of Affective Disorders, 105, $157-165$.

Lincoln, T. M., \& Peters, E. (2019). A systematic review and discussion of symptom specific cognitive behavioural approaches to delusions and hallucinations. Schizophrenia Research, 203, 66-79.

Mazefsky, C. A., Herrington, J., Siegel, M., Scarpa, A., Maddox, B. B., Scahill, L., et al. (2013). The role of emotion regulation in autism spectrum disorder. Journal of the American Academy of Child and Adolescent Psychiatry, 52, 679-688.

Moree, B. N., \& Davis, T. E. (2010). Cognitive-behavioral therapy for anxiety in children diagnosed with autism spectrum disorders: Modification trends. Research in Autism Spectrum Disorders, 4, 346-354.

Morrison, A. P., \& Barratt, S. (2010). What are the components of CBT for psychosis? A delphi study. Schizophrenia Bulletin, 36, 136-142.

Murphy, D., Glaser, K., Hayward, H., Ekland, H., Cadman, T., Findon, J., et al. (2018). Crossing the divide: A longitudinal study of effective treatments for people with autism and attention deficit hyperactivity disorder across the lifespan. Programme Grants for Applied Research, 6, 1-240.

Myhr, G., Talbot, J., Annable, L., \& Pinard, G. (2007). Suitability for short-term cognitive-behavioral therapy. Journal of Cognitive Psychotherapy., 21, 334-345.

National Institute for Health and Care Excellence (NICE). (2011). Autism in under 19 s: Recognition, referral and diagnosis, NICE guidelines [CG128]. London: Department of Health.

National Institute for Health and Care Excellence (NICE). (2012). Autism: Recognition, referral, diagnosis and management of adults on the autism spectrum, NICE guidelines [CG142]. London: Department of Health.

National Institute for Health and Care Excellence NICE. (2011). Common mental health disorders NICE guidelines [CG123]. London: Department of Health.

Nursing and Midwifery Council (NMC). (2015). Professional Standards. England: NMC.

Reaven, J., \& Hepburn, S. (2003). Cognitive-behavioral treatment of obsessive-compulsive disorder in a child with Asperger syndrome. Autism, 7, 145-164.

Renaud, J., Russell, J. J., \& Myhr, G. (2014). Predicting who benefits most from cognitive-behavioral therapy for anxiety and depression. Journal of Clinical Psychology, 70, 924-932.

Rodgers, J., Hodgson, A., Shields, K., Wright, C., Honey, E., \& Freeston, M. (2017). Towards a treatment for intolerance of uncertainty in young people with autism spectrum disorder: Development of the coping with uncertainty in everyday situations (CUESC) programme. Journal of Autism and Developmental Disorders, 47, 3959-3966.

Rossiter, R., \& Holmes, S. (2013). Access all areas: Creative adaptations for CBT with people with cognitive impairments-illustrations and issues. Cognitive Behaviour. Therapist, 6, 4.

Roth, A., \& Piling, S. (2008). Supervision competences framework. London: University College London.

Russell, A. J., Murphy, C. M., Wilson, E., Gillan, N., Brown, C., Robertson, D. M., et al. (2016). The mental health of individuals referred for assessment of autism spectrum disorder in adulthood: A clinic report. Autism, 20, 623-627. 
Safran, J. D., Segal, Z. V., Vallis, T. M., Shaw, B. F., \& Samstag, L. W. (1993). Assessing patient suitability for short-term cognitive therapy with an interpersonal focus. Cognitive Therapy and Research, 17, 23-38.

Scarpa, A., \& Reyes, N. M. (2011). Improving emotion regulation with CBT in young children with high functioning autism spectrum disorders: A pilot study. Behavioural and Cognitive Psychotherapy, 39, 495-500.

Simonoff, E., Pickles, A., Charman, T., Chandler, S., Loucas, T., \& Baird, G. (2008). Psychiatric disorders in children with autism spectrum disorders: Prevalence, comorbidity, and associated factors in a population-derived sample. Journal of the American Academy of Child and Adolescent Psychiatry, 47, 921-929.

Spain, D., Sin, J., Chalder, T., Murphy, D., \& Happe, F. (2015). Cognitive behaviour therapy for adults with autism spectrum disorders and psychiatric co-morbidity: A review. Research in Autism Spectrum Disorders, 9, 151-162.

Thulin, U., Svirsky, L., Serlachius, E., Andersson, G., \& Öst, L.-G. (2014). The effect of parent involvement in the treatment of anxiety disorders in children: A meta-analysis. Cognitive Behaviour Therapy, 43, 185-200.

Tsatsanis, K. (2014). Neuropsychological characteristics of Asperger syndrome. In F. Volkmar, A. Klin, \& J. McPartland (Eds.), Asperger syndrome (pp. 71-102). New York: Guildford Press.

Ung, D., Selles, R., Small, B. J., \& Storch, E. A. (2015). A systematic review and meta-analysis of cognitive-behavioral therapy for anxiety in youth with high-functioning autism spectrum disorders. Child Psychiatry and Human Development, 46, 533-547.

Walters, S., Loades, M., \& Russell, A. (2016). A systematic review of effective modifications to cognitive behavioural therapy for young people with autism spectrum disorders. Review Journal of Autism and Developmental Disorders, 3, 137-153.

Wampold, B. E. (2015). How important are the common factors in psychotherapy? An update. World Psychiatry, 14, 270-277.

Weston, L., Hodgekins, J., \& Langdon, P. E. (2016). Effectiveness of cognitive behavioural therapy with people who have autistic spectrum disorders: A systematic review and meta-analysis. Clinical Psychology Review, 49, 41-54.

Wigham, S., Barton, S., Parr, J. R., \& Rodgers, J. (2017). A systematic review of the rates of depression in children and adults with high-functioning autism spectrum disorder. Journal of Mental Health Research in Intellectual Disabilities, 10, 267-287.

Wigham, S., Rodgers, J., South, M., McConachie, H., \& Freeston, M. (2015). The interplay between sensory processing abnormalities, intolerance of uncertainty, anxiety and restricted and repetitive behaviours in autism spectrum disorder. Journal of Autism and Developmental Disorders, 45, 943-952.

Publisher's Note Springer Nature remains neutral with regard to jurisdictional claims in published maps and institutional affiliations. 